\title{
Caracterización morfológica de hongos endófitos aislados de Hamelia patens Jacq. y Lantana camara L. de Chetumal, Quintana Roo, México
}

\author{
Ángel Salvador Gómez-Rivera* \\ Caribell Yuridia López \\ Maribel Martínez Burgos \\ Xochitl Janett Cachón Mis \\ Instituto Tecnológico de Chetumal
}

\section{Resumen}

Los hongos endófitos son un grupo extenso de organismos que habitan el tejido interno de las plantas y su estudio es una necesidad imperante en la búsqueda de diversidad fúngica en el mundo. En este trabajo se expone la micobiota endofítica asociada con Hamelia patens y Lantana camara mediante la caracterización morfológica de 36 cepas de hongos endófitos (23 organismos aislados de $\mathrm{H}$. patens y 13 de L. camara). Ambas plantas se distinguen por su gran abundancia y amplia distribución en la Península de Yucatán, y se encuentran en la mayoría de las ocasiones en zonas perturbadas y/o en vegetación secundaria. Se obtuvieron en este estudio 24 morfotipos, entre los cuales cuatro no presentaron estructuras reproductivas y fueron considerados como micelio estéril. Para $H$. patens se consignaron 14 morfotipos, siendo los organismos afines al género Acremonium los más frecuentes; por otro lado, para L. camara se registraron diez morfotipos, y predominaron aquellos con estructuras estromáticas o picnidias. Los organismos aislados y caracterizados aquí no representan la diversidad total de hongos endófitos que habitan $H$. patens y L. camara, para ello se requieren trabajos más exhaustivos y un esfuerzo de muestreo aún mayor, sin embargo, esta labor funge como precedente para posteriores investigaciones, no solo en torno a estas dos plantas sino a otros organismos vegetales en las selvas quintanarroenses.

Palabras clave

Endófitos, micobiota, caracterización, vegetación secundaria. 


\title{
Morphological characterization of isolated endophyte fungi from Hamelia patens Jacq. y Lantana camara L. in Chetumal, Quintana Roo, Mexico
}

\author{
Ángel Salvador Gómez-Rivera* \\ Caribell Yuridia López \\ Maribel Martínez Burgos \\ Xochitl Janett Cachón Mis \\ Instituto Tecnológico de Chetumal
}

\begin{abstract}
Endophyte fungi are an extended group of organisms that inhabit the internal plant tissue and the study of them is very important in search of world wide fungal diversity. In this paper the endophytic fungal biota associated with Hamelia patens and Lantana camara through the morphological characterization of 36 strains of endophyte fungi (23 organisms isolated from $H$. patens and 13 from $L$. camara) is presented. Both plants are abundant and widely distributed on the Yucatan Peninsula and are found in disturbed areas as well as in secondary vegetation. 24 morpho-types were found in this study, four had no reproductive structure and were considered sterile mycelium. 14 morpho-types were registered for H. Patens, those organisms related to the Acremonium species were the most frequent. 10 morpho-types were registered for $L$. camara predominantly those with stromal structure. The isolated and characterized organisms presented here do not represent the whole diversity of endophyte fungi that inhabit $H$. patens y L. camara, to do that, a wider sampling effort is needed, nevertheless this effort sets a precedent for further research on these two plants as well as other vegetation in the Quintana Roo rainforest.
\end{abstract}

KEY WORDS

Endophytes, fungal biota, characterization, secondary vegetation.

*E-mail: angel_gomezrivera@hotmail.com 


\section{Introducción}

Los hongos endófitos son microorganismos que habitan, en algún momento de su ciclo vital, el tejido interno de las plantas (Carroll, 1986); su rol ecológico se considera variado pero todavía desconocido (Saikkonen et al., 1998); se les categoriza como patógenos secundarios u organismos comensales, aunque se han demostrado evidencias de mutualismo (Clay, 1988), aunado a su alta producción de metabolitos secundarios importantes en la industria farmacéutica como productos terapéuticos o antibióticos (Schulz et al., 2002; Sánchez-Fernández et al., 2013).

Las descripciones de micobiota endofítica han contribuido a incrementar las estimaciones de diversidad fúngica en el planeta, lo cual ha convertido a los hongos en uno de los grupos de mayor diversidad calculando la existencia de alrededor de 1.5 millones de organismos a escala mundial (Hawksworth, 2001a); en el caso de México se distingue la presencia de 200 mil especies (AguirreAcosta et al., 2014). Aunque ambas cifras en cierta forma se consideran conservadoras, el porcentaje de organismos que se han descrito y estudiado es ínfimo comparado con las cantidades valoradas.

Estudios actuales han demostrado que las zonas tropicales son focos de gran diversidad en cuanto a micromicetos se refiere (Hawskworth, 2001b), encontrando entre estos a los endófitos como un grupo hiperdiverso, y tal diversidad se incrementa mientras más se acerca al ecuador (Arnold et al., 2000; Arnold y Lutzoni, 2007). Se infiere, por lo antes mencionado, que la vegetación tropical representa un depósito inmenso de organismos endófitos sin conocer o caracterizar (Arnold, Maynard y Gilbert, 2001).

El objetivo principal de este estudio es presentar evidencias de la existencia de micobiota endofítica asociada con especies de vegetación secundaria en la ciudad de Chetumal (Quintana Roo, México), mediante el análisis, aislamiento y caracterización morfológica de tales organismos fúngicos. Este esfuerzo de investigación, además de ser el primero en la zona, pretende contribuir al conocimiento de los micromicetos endofíticos que se hallan ocultos en los trópicos, en este caso, vinculados con dos especies de gran abundancia y vasta distribución en la Península de Yucatán: Hamelia patens Jacq. y Lantana camara L.

\section{Antecedentes}

Los hongos endófitos han sido extensamente estudiados en zonas templadas y se ha demostrado un amplio rango de hospederos, encontrándose en plantas de 
Caracterización morfológica de hongos endófitos aislados de Hamelia patens Jacq. y Lantana camara L. de Chetumal, Quintana Roo, México

bosques templados, boreales y tropicales (Stone, Polishook y White, 2004). Las investigaciones de estos organismos en zonas tropicales no son una novedad, aunque, y pese a ello, no son abundantes considerando que esta zona probablemente constituye el refugio más grande de diversidad endofítica en el mundo (Arnold et al., 2000).

En Latinoamérica existen antecedentes de toda índole, desde aquellos estudios enfocados a la ecología y diversidad de la micobiota endófita (Lodge, Fisher y Sutton, 1996; Bayman et al., 1998; Gamboa-Gaitán y Bayman, 2001; Salgado-Salazar y Cepero, 2005), así como las implicaciones que tienen sobre sus hospederos, por ejemplo, en el proceso de fotosíntesis (Gamboa-Gaitán, Laureano y Bayman, 2005) o la actividad antagónica frente a otros microorganismos (Mejía et al., 2008), hasta la búsqueda de metabolitos secundarios de relevancia farmacéutica (Ramírez et al., 2006).

En México hay un acervo pequeño de publicaciones centradas en estos tópicos, no obstante, son de gran valor y utilidad, como el trabajo realizado por Romero, Carrión y Rico-Gray (2001) sobre una especie vegetal medicinal (Parthenium hysterophorus), en el cual se utilizaron siete distintos tipos de esterilización superficial y se encontraron siete géneros de hongos microscópicos (Alternaria, Cylindrocarpon, Curvularia, Fusarium, Nigrospora, Penicillium y Periconia). Otro trabajo importante fue el de Saucedo-García et al. (2014) orientado al estudio de endófitos del café (Coffea arabica) en dos regiones y sistemas de producción agroforestal: se observaron 30 morfoespecies entre las cuales destacan géneros como Alternaria, Beauveria, Colletotrichum, Coniosporium, Cryptopsoriopsis, Diplodia, Glomerella, Guignardia, Paecilomyces, Phomopsis y Xylaria; se advirtió que las plantaciones rústicas albergan una mayor riqueza de morfoespecies, además de que se demuestra que la abundancia de endófitos, riqueza y diversidad pueden verse influenciadas predominantemente por la región de producción y, en menor medida, por el sistema agroforestal.

\section{Metodología}

Se realizó un muestreo no significativo en una zona del sector urbano de la ciudad de Chetumal durante marzo de 2015. Se muestrearon dos individuos por planta distanciados entre sí aproximadamente 20 metros siguiendo el procedimiento de Lodge, Fisher y Sutton (1996) y se recolectó un total de cuatro 
hojas: dos por planta (H. patens y L. camara) que no mostraran síntomas de enfermedad y se distinguieran como maduras o completamente desarrolladas.

Las muestras de material vegetal fueron trasladadas al Laboratorio de Micología, en un tiempo no mayor a 48 horas para su posterior tratamiento que consistió en la esterilización superficial mediante inmersiones en serie en alcohol etílico al $75 \%$ por un minuto, cloro al $50 \%$ por tres minutos (Gamboa-Gaitán y Bayman, 2001) y de nuevo alcohol etílico al $75 \%$ por 30 segundos.

Concluido el proceso de esterilización, el material fue diseccionado hasta conseguir fragmentos menores a $5 \mathrm{~mm}^{2}$ con la finalidad de obtener una mayor eficiencia en el aislamiento (Gamboa-Gaitán, Laureano y Bayman, 2002). Se seleccionaron de manera aleatoria 60 fragmentos (30 por cada planta; 15 por cada hoja), los cuales fueron sembrados en cajas de Petri de 60 x $15 \mathrm{~mm}$ con medio de cultivo de extracto de malta e incubados a $22 \pm 23^{\circ} \mathrm{C}$ en oscuridad total por 30 días.

Finalizado el periodo de crecimiento micelial establecido, los organismos que lograron ser aislados fueron resembrados a medio fresco, para posteriormente llevar a cabo la caracterización mediante la observación de sus estructuras al microscopio en preparaciones frescas con azul lactofenol de anilina. La identificación de los organismos se realizó hasta el nivel taxonómico posible siguiendo las claves dicotómicas pertinentes (Seifert et al., 2011; Williams-Woodward, 2001).

El análisis de datos de la caracterización morfológica se hizo utilizando 156 marcadores morfológicos que representan las variables de evaluación de las características tanto macro como microscópicas, las cuales fueron transformadas a datos binarios de correspondencia y no correspondencia $(0,1)$. Se efectuó un análisis multivariado con conglomerados para distinguir la distancia morfológica existente entre los organismos determinados mediante la representación gráfica de un dendograma con el programa Minitab versión 16.1.0.0. Cabe recalcar que no todos los morfotipos determinados fueron sujetos a este análisis, por ejemplo el caso de los individuos catalogados como micelio estéril debido a la ausencia de estructuras reproductivas, ya que esto alteraría de manera considerable el comportamiento de los datos y, por ende, el dendograma obtenido.

El porcentaje de colonización (PC) y la frecuencia de aislamiento (FA) fueron calculados con las siguientes fórmulas:

$$
P C=\frac{N c}{N t} \times 100 \quad F A=\frac{N i}{N t} \times 100
$$


Caracterización morfológica de hongos endófitos aislados de Hamelia patens Jacq. y Lantana camara L. de Chetumal, Quintana Roo, México

Donde $\mathrm{Nc}$ es el número total de aislamientos, $\mathrm{Ni}$ el total de individuos aislados de un grupo determinado y $N$ el número de fragmentos que fueron sembrados.

\section{Resultados}

Como resultado del aislamiento se registraron 36 organismos de los 60 fragmentos que fueron sembrados. H. patens alcanzó un porcentaje de colonización de $76.67 \%$ (23 individuos de 30 fragmentos sembrados), mientras que L. camara $43.33 \%$ (13 individuos de 30 fragmentos sembrados). Del total de organismos aislados, cuatro no presentaron estructuras reproductivas por lo cual fueron considerados como micelio estéril (tres aislados de $H$. patens y uno de L. camara).

Se obtuvieron 24 morfotipos por medio de la caracterización morfológica de los individuos analizados en todo el estudio; de ellos, tres organismos no pudieron ser determinados o identificados, mientras que el resto fue catalogado en siete grupos taxonómicos.

Cuadro 1. Morfotipos y número de aislamientos en Hamelia patens

\begin{tabular}{|c|c|c|}
\hline \multirow{2}{*}{ MORFOTIPO (DETERMINACIÓN) } & \multicolumn{2}{|c|}{ MUESTRA } \\
\hline & HOJA 1 & HOJA 2 \\
\hline Morfotipo 1 Afín a Acremonium & 1 & 0 \\
\hline Morfotipo 2 Afín a Acremonium & 1 & 1 \\
\hline Morfotipo 3 Coelomycetes & 2 & 1 \\
\hline Morfotipo 4 Phoma & 1 & 0 \\
\hline Morfotipo 5 Curvularia & 2 & 0 \\
\hline Morfotipo 6 Curvularia & 3 & 0 \\
\hline Morfotipo 7 Trichoderma & 1 & 0 \\
\hline Morfotipo 8 Afín a Acremonium & 1 & 0 \\
\hline Morfotipo 9 Micelio estéril & 0 & 3 \\
\hline Morfotipo 10 Afín a Acremonium & 0 & 1 \\
\hline Morfotipo 11 No identificado & 0 & 1 \\
\hline Morfotipo 12 No identificado & 0 & 1 \\
\hline Morfotipo 13 Afín a Acremonium & 0 & 1 \\
\hline Morfotipo 14 Macrophoma & 0 & 2 \\
\hline Número de fragmentos colonizados & 12 & 11 \\
\hline Porcentaje de colonización (Total: 76.67 \%) & $40 \%$ & $36.67 \%$ \\
\hline
\end{tabular}

Fuente: Elaboración propia. 
Para $H$. patens se consignaron 14 morfotipos, cinco de los cuales presentan alta afinidad al género Acremonium, dos corresponden al género Curvularia, uno a cada uno de los géneros Phoma, Macrophoma y Trichoderma, y uno a la clase Coelomycetes. Dos organismos no pudieron identificarse ni ubicarse en algún grupo y un individuo fue señalado como micelio estéril (cuadro 1).

Los aislamientos de L. camara dieron como resultado diez morfotipos, cinco de los cuales correspondieron a la clase Coelomycetes. Los géneros Sphaeropsis, Curvularia y Acremonium presentaron un morfotipo cada uno, además de encontrarse un organismo considerado como micelio estéril y uno más que no pudo ser identificado (cuadro 2).

Cuadro 2. Morfotipos y número de aislamientos en Lantana camara

MORFOTIPO (DETERMINACIÓN)

\begin{tabular}{|c|c|c|}
\hline & \multicolumn{2}{|c|}{ MUESTRA } \\
\hline & HOJA 1 & HOJA 2 \\
\hline & 1 & 0 \\
& 1 & 0 \\
& 1 & 1 \\
& 1 & 1 \\
& 1 & 0 \\
& 0 & 1 \\
& 1 & 0 \\
\hline & 2 & 0 \\
\hline & 1 & 0 \\
\hline & 10 & 0 \\
\hline
\end{tabular}

Fuente: Elaboración propia.

Como resultado de la caracterización morfológica mediante el análisis estadístico por conglomerados, se obtuvo un dendograma basado en los morfotipos determinados que exhibe el grado de distancia entre ellos (figura 1). 
Caracterización morfológica de hongos endófitos aislados de Hamelia patens Jacq. y Lantana camara L. de Chetumal, Quintana Roo, México

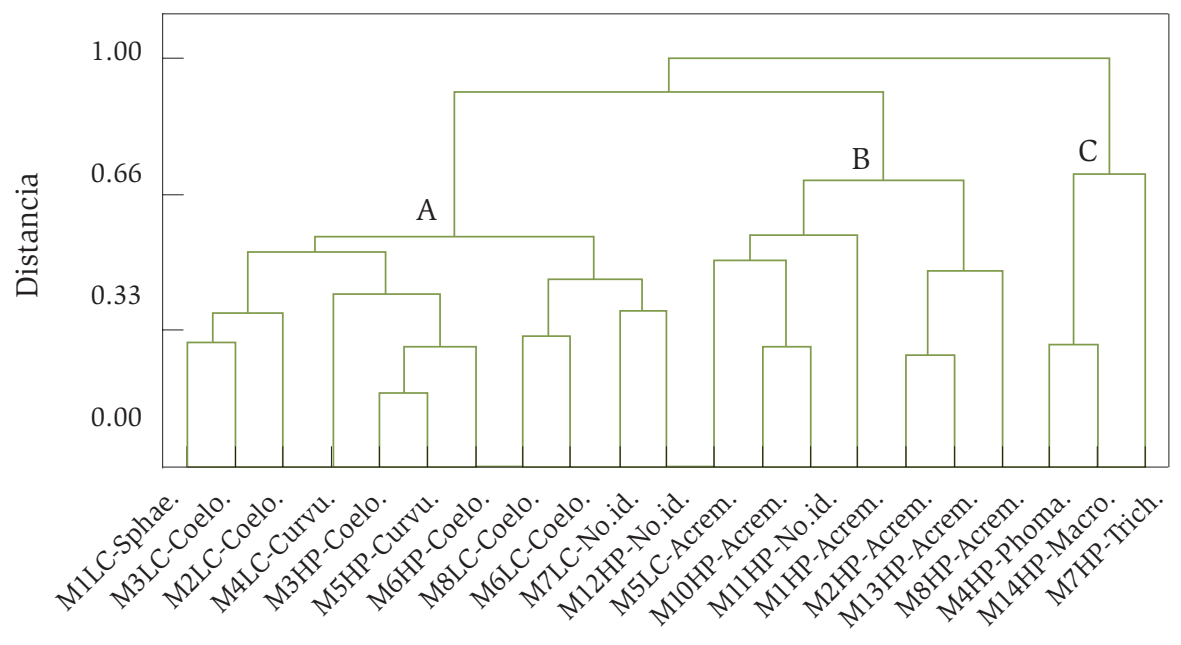

Morfotipos determinados

Fuente: Elaboración propia.

Figura 1. Caracterización morfológica de los organismos determinados

\section{Discusión}

Los hongos endófitos determinados en el presente estudio hasta nivel genérico representan en su mayoría a taxones previamente registrados como endófitos en zonas tropicales (Wanderley-Costa et al., 2012), a excepción de los géneros Macrophoma y Sphaeropsis, no obstante, la determinación taxonómica mostrada en este trabajo requiere de su confirmación llevando a cabo análisis bioquímicos y moleculares.

Los porcentajes de colonización resultantes fueron bajos en comparación con los reportados en otros estudios en zonas tropicales del continente americano, donde se han obtenido porcentajes por encima de 90 \% (Lodge, Fisher y Sutton, 1996; Gamboa-Gaitán y Bayman, 2005; Gamboa-Gaitán, Laureano y Bayman, 2002). Lo anterior puede deberse al tamaño de la muestra, que, como se mencionó al principio del trabajo, no fue significativa, o a que las plantas muestreadas se hallaban en un área perturbada dentro de la ciudad de Chetumal, en cuyo caso es remarcable la existencia de estos organismos en zonas con alto grado de impacto 
antropogénico, ya que probablemente la presencia de hongos endófitos es mayor mientras más conservada se encuentre la vegetación (Gamboa-Gaitán y Bayman, 2001; Rodriges, 1994 cit. en Gamboa-Gaitán y Bayman, 2001; Salgado-Salazar y Cepero, 2005).

El género Acremonium fue el organismo más frecuente para $H$. patens, sin embargo, este taxón manifiesta un problema en su identificación y debe tomarse con reservas dado que hay docenas de géneros afines a este y es solo una pequeña diferencia en los tamaños de las estructuras reproductivas lo que podría llevar la determinación a un género distinto.

Por otro lado, para L. camara el grupo taxonómico más recurrente en el aislamiento fue la clase Coelomycetes que agrupa un sinnúmero de taxones con estructuras estromáticas como picnidias o acérvulos, lo que justifica de algún modo que estos hayan sido los organismos más asiduos en los aislamientos para esta planta. Cabe recalcar que, curiosamente, los morfotipos con estructuras estromáticas (Coelomycetes) ocuparon casi la totalidad de los individuos aislados para L. camara, pudiendo existir una relación entre estos y su hospedero, aunque son necesarios estudios posteriores para confirmar o refutar esta aseveración.

En el dendograma resultante de la caracterización morfológica (figura 1) se puede observar la presencia de tres grandes grupos de individuos A, B y C, siendo el último el más distante con respecto a los dos primeros debido a que los morfotipos que se agrupan en él no mostraron estructuras de resistencia del tipo clamidosporas. El conglomerado A representa a todos los organismos con estructuras estromáticas o picnidios que es una característica usada para distinguir a los hongos del grupo de los Coelomycetes, y, por último, el conglomerado B está constituido por organismos afines a los hifomicetos.

El conglomerado B pertenece a morfotipos aislados de $H$. patens, a excepción del morfotipo 5 de L. camara determinado como afín a Acremonium; por otra parte, el grupo A pertenece en su mayoría a morfotipos de L. camara, salvo los morfotipos 6, 8 y 12 de $H$. patens.

Los individuos que exhibieron mayor similitud entre ellos, de acuerdo con su morfología, fueron los morfotipos 3 (Coelomycetes) y 5 (Curvularia) de $H$. patens, y el más distante fue el morfotipo del género Trichoderma perteneciente de igual manera a $H$. patens que se encuentra en el conglomerado $\mathrm{C}$ dado que presentó estructuras únicas que no se mostraron en los demás morfotipos. 
Caracterización morfológica de hongos endófitos aislados de Hamelia patens Jacq. y Lantana camara L. de Chetumal, Quintana Roo, México

\section{Conclusiones y comentarios finales}

Cuando se llevan a cabo estudios de micromicetos, son necesarias descripciones exhaustivas y detalladas para lograr identificar ciertos géneros y aún más si lo que se busca es la determinación taxonómica en el nivel de especie. Las determinaciones presentadas en este trabajo requieren, como ya se ha indicado, de la confirmación taxonómica mediante estudios moleculares; más aún aquellos organismos que no produjeron ninguna estructura reproductiva que los distinga precisan de estos para ser identificados.

Debido a la naturaleza preliminar del estudio es probable que los organismos aislados y caracterizados aquí no representen la diversidad total de hongos endófitos que habitan $H$. patens y L. camara, ya que para conseguirlo es menester un trabajo de laboratorio aún más exhaustivo y un esfuerzo de muestreo mayor. Sin embargo, los resultados obtenidos en esta investigación constituyen un importante aporte para construir las bases de estudios posteriores, no solo de estas dos plantas, sino para otros organismos vegetales en las selvas quintanarroenses.

Como comentario final, cabe destacar que el estudio de los hongos endófitos en zonas tropicales debe ser considerado una prioridad en el conocimiento y conservación de la biodiversidad, debido tanto al amplio potencial de uso, como a las amenazas que enfrentan los ecosistemas vegetales. Los resultados que podrían obtenerse al investigar los endófitos podrían aplicarse a sectores productivos como el agroforestal y el farmacéutico.

\section{Fuentes consultadas}

Aguirre-Acosta, E., M. Ulloa, S. Aguilar, J. Cifuentes y R. Valenzuela (2014). "Biodiversidad de hongos en México". Revista Mexicana de Biodiversidad, 85, 76-81.

Arnold, A. E. y F. Lutzoni (2007). "Diversity and host range of foliar fungal endophytes: are tropical leaves biodiversity hotspots?”. Ecology, 88 (3), 541-549.

Arnold, A. E., Z. Maynard y G. S. Gilbert (2001). "Fungal endophytes in dicotyledonous neotropical trees: patterns of abundance and diversity". Mycological Research, 105 (12), 1502-1507.

Arnold, A. E., Z. Maynard, G. S Gilbert, P. D. Coley y T. A. Kursar (2000). “Are tropical fungal endophytes hiperdiverse?”. Ecology Letters, 3, 267-274. 
Bayman, P., P. Angulo-Sandoval, Z. Baez-Ortiz y D. J. Lodge (1998). “Distribution and dispersal of Xylaria endophytes in two tree species in Puerto Rico”. Mycological Research, 102 (8), 944-948.

Carroll, G. C. (1986). "The biology of endophytism in plants with particular reference to woody perennials”, en N. J. Fokkema y J. Van den Heuvel (eds.). Microbiology of the Phyllosphere. Cambridge: Cambridge University Press, 205-222.

Clay, K. (1988). "Fungal endophytes of grasses: a defensive mutualism between plants and fungi”. Ecology, 69, 10-16.

Gamboa-Gaitán, M. A. y P. Bayman (2001). "Communities of Endophytic Fungi in Leaves of a Tropical Timber Tree (Guarea guidonia: Meliaceae)". Biotropica, 33 (2), 352-360.

Gamboa-Gaitán, M. A., S. Laureano y P. Bayman (2002). “Measuring diversity of endophytic fungi in leaf fragments: Does the size matter?”. Mycopathologia, 156, 41-45.

Gamboa-Gaitán, M. A., S. Laureano y P. Bayman (2005). "Endophytic Phomopsis Strains from leaves of Guarea Guidonia (Meliaceae)”. Caribbean Journal of Science, 41 (2), 215-224.

Hawksworth, D. L. (2001a). "The magnitude of fungal diversity: the 1.5 million species estimate revisited”. Mycological Research, 105 (12), 1422-1432.

Hawksworth, D. L. (2001b). "Why study tropical fungi?”, en R. Watling, J. C. Frankland, A. M. Ainsworth, S. Isaac y C. H. Robinson (eds.). Tropical Mycology: Vol. 2, Micromycetes. San Diego: Elsevier Academic Press, 241-270.

Lodge, D. J., P. J. Fisher y B. C. Sutton (1996). "Endophytic Fungi of Manilkara bidentata leaves in Puerto Rico”. Mycologia, 88 (5), 733-738.

Mejía, L. C., E. I. Rojas, Z. Maynard, S. VanBael, A. E. Arnold, H. Prakash, G. J. Samuels, N. Robbins y E. Allen-Herre (2008). "Endophytic fungi as biocontrol agents of Theobroma cacao pathogens". Biological Control, 43, 4-14.

Ramírez, J. Y., E. Delgado-Fernández, M. Rodolfi y T. Solveig (2006). “Actividad antagónica de hongos endófitos de plantas medicinales del Ecuador sobre bacterias patógenas”. Boletín Micológico, 21, 49-53.

Romero, A., G. Carrión y V. Rico-Gray (2001). "Fungal latent pathogens and 
Caracterización morfológica de hongos endófitos aislados de Hamelia patens Jacq. y Lantana camara L. de Chetumal, Quintana Roo, México

endophytes from leaves of Parthenium hysterophorus (Asteraceae)". Fungal Diversity, 7, 81-87.

Saikkonen, K., S. H. Faeth, M. Helander y T. J. Sullivan (1998). "Fungal endophytes: a continuum of interactions with host plants". Annual Review of Ecology and Systematics, 29, 319-343.

Salgado-Salazar, C. y M. C. Cepero (2005). “Aislamiento de hongos endófitos en rosa (Rosa hybrida) en Bogotá, Colombia”. Revista Iberoamericana de Micología, 22, 99-101.

Sánchez-Fernández, R. E., B. L. Sánchez-Ortiz, Y. K. M. Sandoval-Espinosa, A. Ulloa-Benítez, B. Armendáriz-Guillén, M. C. García-Méndez y M. L. Macías-Rubalcava (2013). "Hongos endófitos: fuente potencial de metabolitos secundarios bioactivos con utilidad en agricultura y medicina”. Revista Especializada en Ciencias Químico-Biológicas, 16 (2), 132-146.

Saucedo-García, A., A. L. Anaya, F. J. Espinosa-García y M. C. Gonzáles (2014). "Diversity and Communities of Foliar Endophytic Fungi from Different Agroecosystems of Coffea arabica L. in Two Regions of Veracruz, Mexico”. PLos ONE, 9 (6), e98454.

Schulz, B., C. Boyle, S. Draeger, A. Rommert y K. Krohn (2002). "Endophytic fungi: a source of novel biologically active secondary metabolites". Mycological Research, 106, 996-1004.

Seifert, K., G. Morgan-Jones, W. Gams y B. Kendrick (2011). The Genera of Hyphomycetes. Utrecht: CBS Biodiversity.

Stone, J. K., J. D. Polishook y J. F. White (2004). “Endophytic Fungi”, en G. M. Muller, G. F. Bills y M. S. Foster (eds.). Biodiversity of Fungi: Inventory and Monitoring Methods. San Diego: Elsevier Academic Press, 241-270.

Wanderley-Costa, I. P., M. M. Costa-Assuncao, T. E. Félio, R. J. Vivela y M. A. Queiroz-Cavalcanti (2012). "Cheklist of endophytic from tropical regions”. Mycotaxon, 119-494.

Williams-Woodward, J. (2001). Simplified fungi identification key [en línea]. Georgia: The University of Georgia-Cooperative Extension Service-College of Agricultural \& Environmental Sciences. Disponible en: http:// plantpath.caes.uga.edu/extension/documents/fungikey.pdf 